\title{
A Simulink Model for Damping Power System Oscillations Using Fact Devices
}

\author{
B.Ramu ${ }^{1}$, K. Ashwini ${ }^{2}$, K. Damodara Reddy ${ }^{3}$, Md. Asif ${ }^{4}$, P.Harika ${ }^{5}$ \\ 1,2,3,4,5 (EEE, Vardhaman College of Engineering (Autonomous), JNTU, Hyderabad, India)
}

\begin{abstract}
A new control schème for damping power system oscillations using fact devices is employed instead of the traditional firing angle control schème. In this paper the effectiveness of fact devices in damping power system oscillations is evaluated. Fact devices are SVC(static var compensator),TCSC(thyristor controlled series capacitor),DFC(dynamic power flow controller),STATCOM(static synchronous compensator),SSSC(static series synchronous compensator),UPFC(unified power flow controller). The most commonly used fact devices for damping power system oscillations are TCSC,SSSC. The impact of the SVR controlled TCSC on SSR is investigated in this real-time simulator by analyzing the system damping.
\end{abstract}

Keywords: FACTS Controllers, phase imbalance, series compensation, thyristor controlled series capacitor.

\section{INTRODUCTION}

FACTS Controllers provide the flexibility of controlling both real and reactive power which could result in an excellent capability for improving power system dynamics. flexible AC Transmission Systems (FACTS) technology provides unprecedented way for controlling transmission grids and increasing transmission capacity. series compensation is an effective mean to increase the electrical power transfer capability of highvoltage transmission lines.

Insertion of a capacitive reactance in series with the line's inherent inductive reactance lowers the total, effective impedance of the line and thus virtually reduces its length. As a result both angular and voltage stability in the power system gets improved. A side effect of the inserting a series capacitor in series with the transmission line is that an electrical resonance will be introduced. In the 70'ies it was experienced that this electrical resonance may be harmful if a series compensated transmission line is connected electrically close to a thermal power station. The reason is that the shaft system joining the electrical generator with the various turbine stages exhibits mechanical torsional resonance at various "sub synchronous" frequencies, i.e. frequencies below the nominal 50 or $60 \mathrm{~Hz}$ frequency of the network. At certain unfavorable relations between the electrical and mechanical resonance frequencies oscillations with exponentially increasing amplitude can be excited spontaneously. This condition is being referred to as a Torsional Interaction Sub Synchronous Resonance "TI-SSR". This condition potentially may cause damage to the generator with severe consequences for the power supply and causing harsh economical loss.

A problem of interest in the power industry at which FACTS Controllers could play a significant role in it is increasing damping of low frequency power oscillations that often arise between areas in large interconnected power networks. These oscillations are termed inter-area oscillations, which are normally characterized by poor damping . Inter-area oscillations can severely restrict system operations by requiring the curtailment of electric power transfers level as an operational measure. These oscillations can also lead to widespread system disturbances. Several studies have investigated the potential of using FACTS Controllers' capability in damping inter-area oscillations. The use of Thyristor Controlled Series Capacitor (TCSC), and Static Synchronous Series Compensator (SSSC) have been the subjects of several studies evaluating their respective effectiveness in enhancing power system dynamics.

\section{Modeling Of The Single Phase Tcsc}

The recently proposed phase imbalanced series capacitive compensation concept has been shown to be effective in enhancing power system dynamics as it has the potential of damping power swing as well as sub synchronous resonance oscillations. Fig. 1 shows a scheme for a phase imbalanced capacitive compensation. It is a "hybrid" series compensation scheme, where the series capacitive compensation in one phase is created using a single-phase TCSC in series with a fixed capacitor (Cc), and the other two phases are compensated by fixed series capacitors (C). The TCSC control is initially set such that its equivalent compensations at the power frequency combined with the fixed capacitor yield a resultant compensation equal to the other two phases. Thus, the phase balance is maintained at the power frequency while at any other frequency, a phase imbalance is created. To further enhance power oscillations damping, the TCSC is equipped with a supplementary controller. 


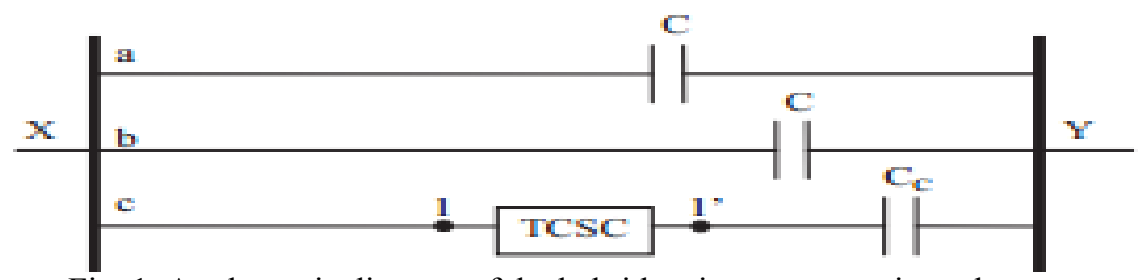

Fig. 1. A schematic diagram of the hybrid series compensation scheme

The phase imbalance of the proposed scheme can be explained mathematically as follows:

1) At the power frequency, the series reactances between buses $X$ and $Y$, in Fig. 1, in phases a, b, and c are given by:

$$
\begin{aligned}
& X_{a}=X_{b}=\frac{1}{j \omega_{o} C} \\
& X_{c}=\frac{1}{j \omega_{o} C_{c}}-j X_{\text {TCSCo }}
\end{aligned}
$$

where $-\mathrm{j} \mathrm{X}_{\mathrm{TCSC}}$ is the effective capacitive reactance of the TCSC at the power frequency such that $\mathrm{Xa}=\mathrm{Xb}=\mathrm{Xc}$

2) During any other frequency, $f$

$$
X_{c}=\frac{1}{j \omega_{e} C_{c}}-j X_{T C S C o}-j \Delta X_{T C S C}
$$

The first terms in (2) and (3) are different because of the difference in frequency. The third term in (3) represents the change in the effective capacitive reactance of the TCSC due to the action of the TCSC supplementary controller. This scheme would, definitely, be economically attractive when compared with a full three-phase TCSC which has been used/proposed for power oscillations damping. Furthermore, reducing the number of thyristor valves to one third will also have a positive impact on system reliability.

The effectiveness of the scheme in damping power swings and sub synchronous resonance oscillations is reported in. This paper evaluates the effectiveness of the scheme in damping power system oscillations.

To demonstrate the effectiveness of the proposed scheme in power system oscillations damping, the system shown in Fig. 2 is adopted as a test benchmark. It consists of three large generating stations (G1, G2 and G3) supplying two load centers (S1 and S2) through five $500 \mathrm{kV}$ transmission lines. The two double-circuit transmission lines L1 and L2 are series compensated with fixed capacitor banks located at the middle of the lines. The compensation degree of $\mathrm{L} 1$ and $\mathrm{L} 2$ is $50 \%$. The compensation degree is defined as the ratio $(\mathrm{XC} / \mathrm{XL})^{*} 100 \%$ for fixed capacitor compensated phases and $\left(\mathrm{X}_{\mathrm{Cc}}+\mathrm{X}_{\mathrm{TCSC}}\right) / \mathrm{XL}^{*} 100 \%$ for the hybrid compensated phase.

The total installed capacity and peak load of the system are 4500 MVA and 3833 MVA respectively. Shunt capacitors are installed at buses 4 and 5 to maintain their voltages within $1 \pm 0.05$ p.u. Two loading profiles designated as Load Profiles A and B are considered in the investigations of this paper. In Load Profile A, S1 = $1400+\mathrm{j} 200$ MVA and S2 $=2400+\mathrm{j} 300$ MVA while in Load Profile B, S1 $=2000+\mathrm{j} 200 \mathrm{MVA}$ and S2 $=1800$ $+\mathrm{j} 300 \mathrm{MVA}$. The power flow results for the bus voltages and the line real power flows of the system for these two loading profiles are shown in the Appendix.

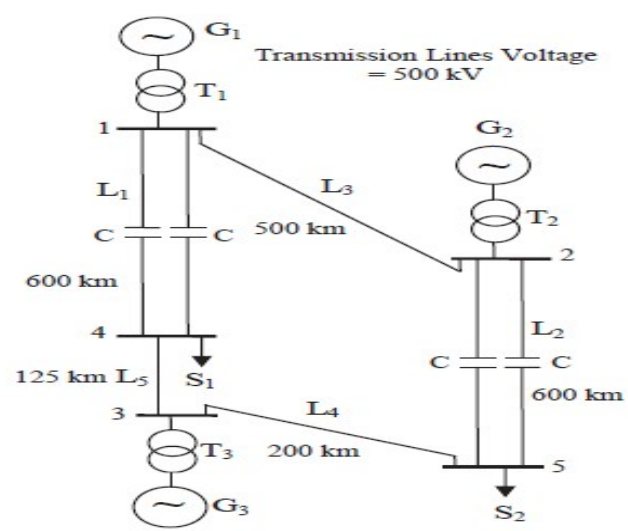

Fig. 2. Test benchmark. 
The single-phase TCSC is modeled in the EMTP-RV as a single module using an ideal thyristor pair and an RC snubber circuit as shown in Fig. 3. A Phase Locked Loop (PLL) is used to extract phase information of the fundamental frequency line current, which will be used to synchronize TCSC operation. The thyristor gating control is based on the Synchronous Voltage Reversal (SVR) technique. The TCSC impedance is measured in terms of a boost factor $k B$, which is the ratio of the apparent reactance of the TCSC seen from the line to the physical reactance of the TCSC capacitor bank. A positive value of $k B$ is considered for capacitive operation. A low-pass filter based estimation algorithm is used to estimate the voltage and the current phasors. A boost measurement block performs complex impedance calculations for the boost factor of the TCSC as $k B=$ Imag $\square V^{\wedge} C / I^{\wedge} C \square / X_{C T C S C}$, where, $V^{\wedge} C$ and $I^{\wedge} C$ are the estimated phase voltage and current and $X_{C T C S C}$ is the capacitive reactance of the TCSC capacitor branch at the fundamental frequency. A proportional-integral (PI) control based boost level controller is implemented to control the TCSC boost level to the desired value by adjusting the instant of the expected capacitor voltage zero crossing. The integral part of the controller helps in removing the steady state errors. The controller parameters were determined by performing repeated time domain simulations for the different operating conditions. This algorithm uses the difference between the actual boost level and the reference boost level (err) shown in Fig. 3 as an objective function. The algorithm starts with arbitrary initial values for the control parameters and calculates the values of the objective function each time. The control parameters are incremented for the next iteration and the procedure is repeated until the objective function approaches a minimum value (below a threshold value). The procedure described above is widely used by industry for tuning of controller parameters.

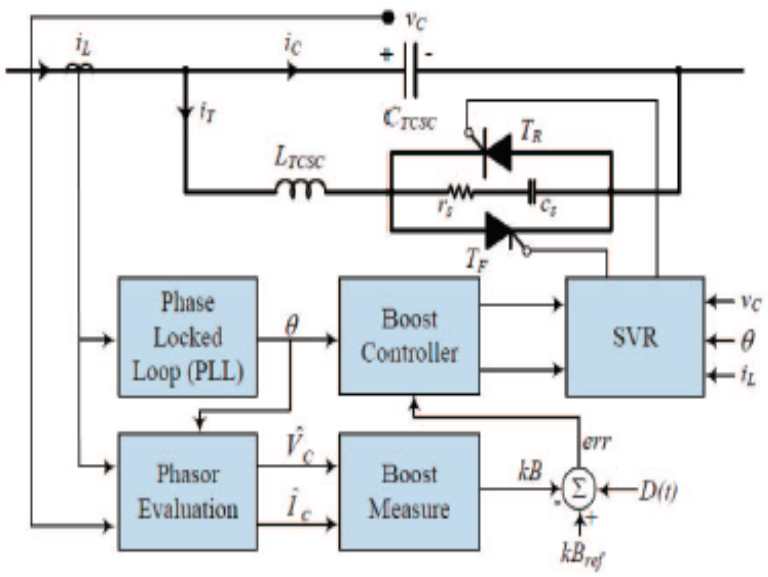

Fig. 3. Block diagram of a TCSC controller

In Fig. 3, $\mathrm{D}(\mathrm{t})$ is a supplemental signal generated from an $m$-stage lead-lag compensation based controller. As the real power flow in the transmission line is proportional to the inverse of the total line reactance, the power swing damping can be achieved by properly modulating the apparent TCSC reactance through this controller. The supplemental controller input (stabilizing) signals could be local (e.g. real power flows) or remote (e.g. load angles or speed deviations of remote generators). If a wide-area network of Synchronized Phasor Measurement (SPM) units is available, then the remote signals can be downloaded at the controller in real time without delay. Local signals are generally preferred over remote signals as they are more reliable since they do not depend on communications.

\subsection{Load profile A}

\section{Case Studies}

In this case, four different combinations of stabilizing signals (tabulated in Table I) are examined in order to determine the combination that would result in the best system transient time responses. The final results of the time-domain simulation studies (controllers tuning) are shown in Fig. 5 which illustrates the generator load angles, measured with respect to generator 1 load angle, during and after fault clearing. The transfer functions of the TCSC supplemental controllers for the four combinations are given in Table II. Comparing the responses of the fixed series capacitor compensation to the hybrid TCSC compensation scheme in Fig. 5, the positive contribution of the proposed hybrid scheme to the damping of the system oscillations is very clear. As it can be seen from Fig. 5, the power swing damping controller effectively damps the system oscillations. It can also be seen from Fig. 5 that the best damping of the relative load angle responses are achieved with the del21-del21 combination. The second best damped responses are obtained with the del31del21 combination. These results should be expected due to the direct relationship between the relative load 
angles and the generators that yield the problem. It can also be seen from Fig. 5 that the worst damped responses are obtained with PL1- del21 combination which results also in the increase of the first swings

\section{TABLEI}

The Four Examingd Combnations of Stabuzizng Stgnals for CASE

STODYI

\begin{tabular}{|c|c|c|}
\hline Combination & Each TCSC in $\mathrm{L}_{1}$ & Each TCSC in $\mathrm{L}_{2}$ \\
\hline 1 & $\delta_{21}$ & $\delta_{21}$ \\
\hline 2 & $\delta_{31}$ & $\delta_{21}$ \\
\hline 3 & $\delta_{31}$ & $\mathrm{P}_{\mathrm{L} 2}$ \\
\hline 4 & $\mathrm{P}_{\mathrm{L} 1}$ & $\delta_{21}$ \\
\hline
\end{tabular}

TABLE II

Transfer Functions of the TCSC SUPPLEMENTAL CONTROLIERS For

CASE STUDY I

\begin{tabular}{|c|c|c|}
\hline $\begin{array}{c}\text { Combi- } \\
\text { nation }\end{array}$ & Each TCSC in $\mathrm{L}_{1}$ & Each TCSC in $\mathrm{L}_{2}$ \\
\hline 1 & $G(s)=0.25 \frac{10}{(s+10)} \frac{3 s}{(3 s+1)}$ & $G(s)=-0.15 \frac{10}{(s+10) \frac{3 s}{(3 s+1)}}$ \\
\hline 2 & $G(s)=0.05 \frac{10}{(s+10) \frac{3 s}{(3 s+1)}}$ & $G(s)=-0.15 \frac{10}{(s+10) \frac{3 s}{(3 s+1)}}$ \\
\hline 3 & $G(s)=0.1 \frac{10}{(s+10)} \frac{3 s}{(3 s+1)}$ & $G(s)=-0.4 \frac{10}{(s+10) \frac{3 s}{(3 s+1)}}$ \\
\hline 4 & $G(s)=-0.25 \frac{10}{(s+10)} \frac{3 s}{(3 s+1)}$ & $G(s)=-0.25 \frac{10}{(s+10)} \frac{3 s}{(3 s+1)}$ \\
\hline
\end{tabular}

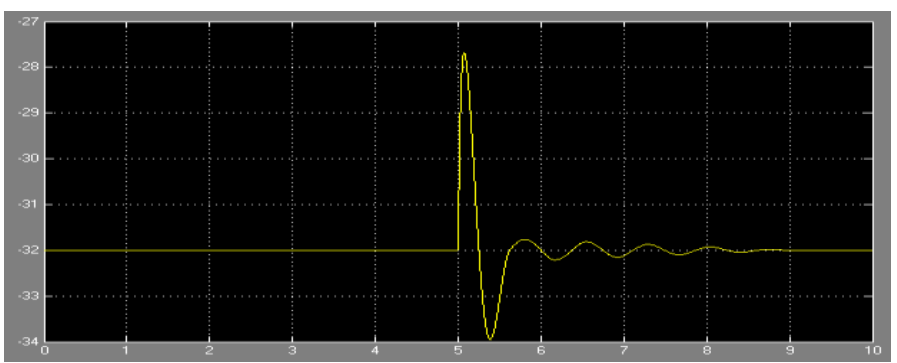

Fig 4: Fixed c

Hssc_d31d21_A:

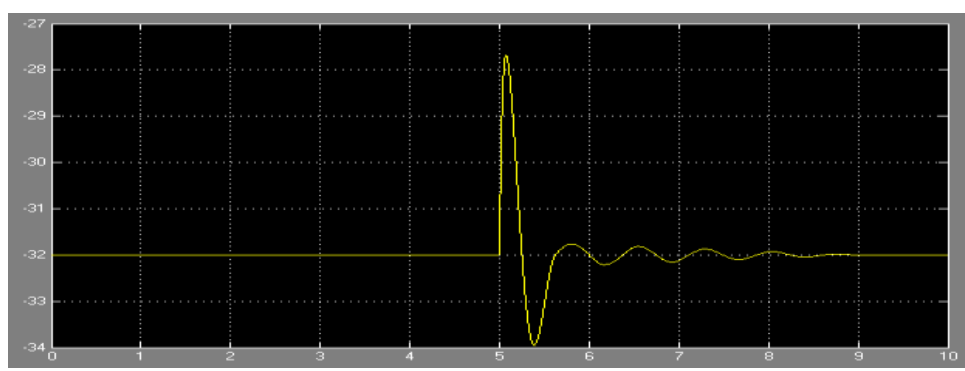

FIG 5: DEL21

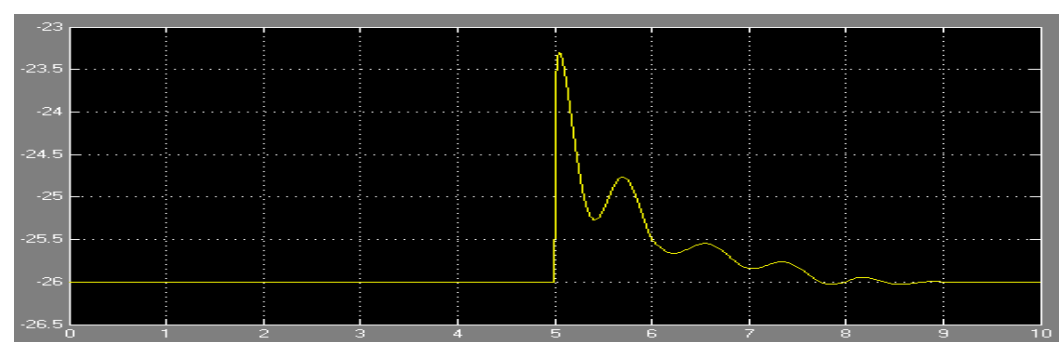

Fig 6 : DEL31 
hssc_d31PL2_A

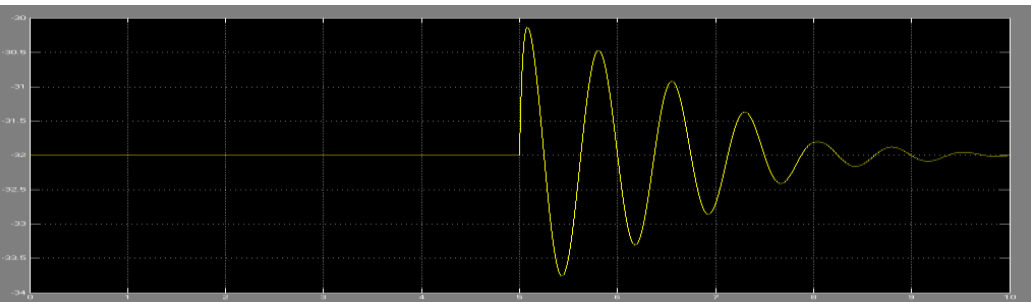

Fig 7 : DEL21

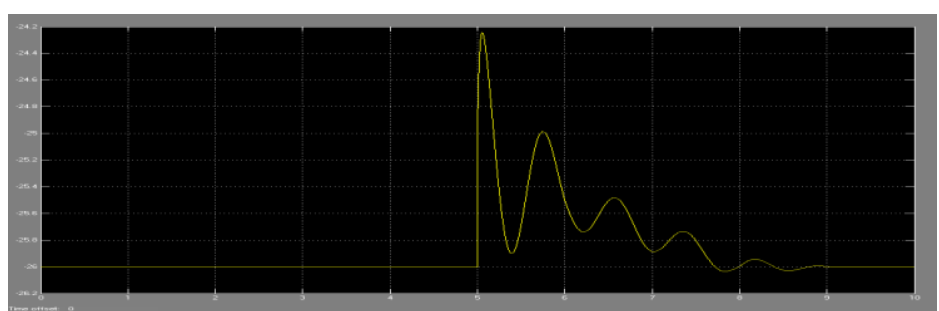

Fig 8 : DEL31

\subsection{Load profile B}

In this case, del21 is used as the supplementary controllers stabilizing signal. The transfer functions of the TCSC supplemental controllers are given in Table III. Fig. 6 illustrates the generator load angles, measured with respect to generator 1 load angle, during and after fault clearing. It can be seen from Fig. 6 that, at this loading profile, the hybrid single-phase-TCSC scheme provides again a better damping performance to system oscillations compared to fixed capacitor compensation. It is observed, however, that there is a slight increase in the first swing of del 21 .

TABLE III

Transfer FunCtIONS OF THE TCSC SUPPLEMENTAL CONTROII ERS FOR CASE STUDY II

\begin{tabular}{|l|l|}
\hline Each TCSC in $\mathrm{L}_{1}$ & $G(s)=0.3 \frac{10}{(s+10) \frac{3 s}{(3 s+1)}}$ \\
\hline Each TCSC in $\mathrm{L}_{2}$ & $G(s)=-0.15 \frac{10}{(s+10)} \frac{3 s}{(3 s+1)}$ \\
\hline
\end{tabular}

hssc_fixedcompensation_B

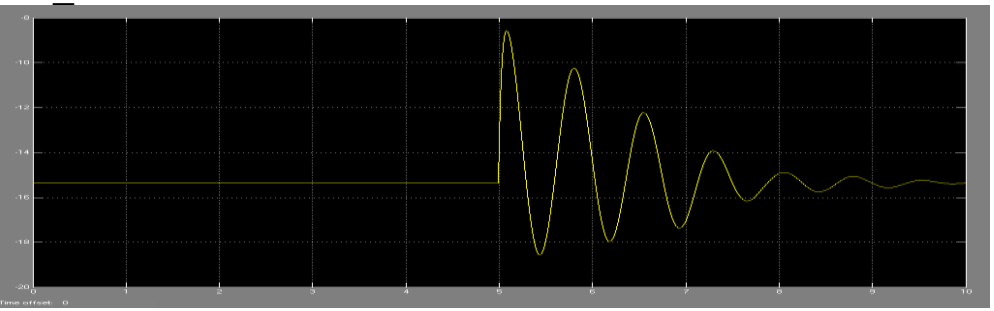

Fig 9:

Hssc_d21d21_B

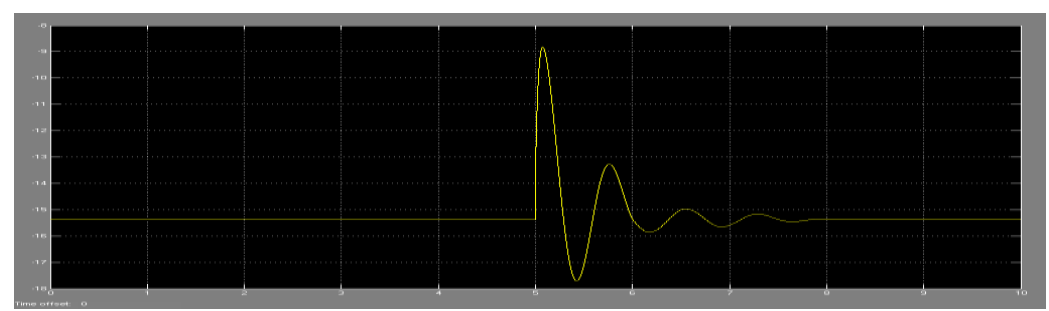

Fig 10: Del 21 


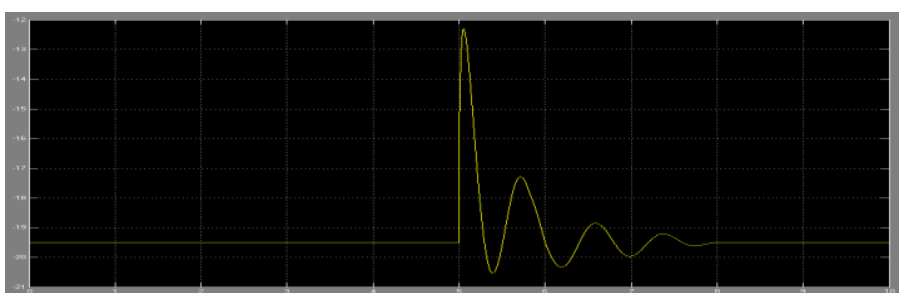

Fig 11: Del31

\section{CONCLUSION}

The paper presents the application of fact devices in damping power system oscillations. The effectiveness of the presented scheme in damping these oscillations is demonstrated through several digital computer simulations of case studies on a test benchmark. The presented hybrid series capacitive compensation scheme is feasible, technically sound, and has an industrial application potential.

\section{REFERENCES}

[1] Narain G. Hingorani and Laszlo Gyugyi, "Understanding FACTS, Concepts and Technology of Flexible AC Transmission Systems," IEEE Press, 2000

[2] M. Klein, G.J. Rogers and P. Kundur, “A Fundamental Study of Inter-Area Oscillations in Power Systems,” IEEE Transactions on Power Systems, Vol. 6, No. 3, 1991, pp. 914-921.

[3] E.V. Larsen, J.J. Sanchez-Gasca and J.H. Chow, "Concepts for Design of FACTS Controllers to Damp Power Swings," IEEE Transactions on Power Systems, Vol. 10, No. 2, May 1995, pp. 948-956.

[4] B. Chaudhuri, B. Pal, A. C. Zolotas, I. M. Jaimoukha, and T. C. Green, "Mixed-sensitivity Approach to H Control of Power System Oscillations Employing Multiple FACTS Devices,” IEEE Transactions on Power System, Vol. 18, No. 3, August 2003, pp. 11491156.

[5] B. Chaudhuri and B. Pal, "Robust Damping of Multiple Swing Modes Employing Global Stabilizing Signals with a TCSC," IEEE Transactions on Power System, Vol. 19, No. 1, February 2004, pp. 499-506.

[6] R. Majumder, B.C. Pal, C. Dufour and P. Korba, "Design and Real-Time Implementation of Robust FACTS Controller for Damping Inter-Area Oscillation," IEEE Transactions on Power Systems, Vol. 21, No.2, May 2006, pp. 809-816.

[7] D. Rai, G. Ramakrishna, S.O. Faried and A. Edris," Enhancement of Power System Dynamics Using a Phase Imbalanced Series Compensation Scheme," IEEE Transactions on Power Systems, Vol. 25, No. 2, May 2010, pp. 966-974.

[8] H. Xie and L. Ängquist, "Synchronous Voltage Reversal control of TCSC - impact on SSR conditions," Proceedings of the Nordic Workshop on Power and Industrial Electronics (NORPIE), 2004.

[9] Lennart Ängquist, "Synchronous Voltage Reversal Control of Thyristor Controlled Series Capacitor," Royal Institute of Technology, TRITAETS- 2002-07, ISSN 1650-674X. 